\title{
The value of global longitudinal strain and galectin-3 for predicting cardiovascular events in patients with severe aortic stenosis
}

\author{
Lucia Agoston-Coldea ${ }^{1}$, Kunal Bheecarry ${ }^{1}$, Cristian Petra ${ }^{1}$, Lelia Strambu ${ }^{2}$, Camelia Ober ${ }^{2}$, \\ Radu Revnic ${ }^{1}$, Silvia Lupu ${ }^{3}$, Teodora Mocan ${ }^{4}$, Daniela Fodor ${ }^{1}$
}

${ }^{1} 2^{\text {nd }}$ Department of Internal Medicine, "Iuliu Hatieganu" University of Medicine and Pharmacy, Cluj-Napoca, 2 "Niculae Stancioiu" Heart Institute, Cluj-Napoca, ${ }^{3}$ Department of Physiology, University of Medicine and Pharmacy of Târgu Mures, ${ }^{4}$ Department of Physiology, "Iuliu Hatieganu" University of Medicine and Pharmacy, Cluj-Napoca, Romania

\begin{abstract}
Aims: Left ventricular global longitudinal strain (GLS) was shown to predict outcomes after valve replacement in patients with aortic stenosis (AS). In the current study, we aimed to test the combined use of GLS and a marker of fibrosis - Galectin-3 - for predicting major cardiovascular adverse events (MACEs) in patients with severe AS. Materials and methods: We conducted a prospective study on 42 patients with severe AS and 42 volunteers. Patient evaluation included biochemistry tests, electrocardiogram, 24-hour Holter monitoring, the 6-minute walk test, and echocardiography. Outcomes of AS patients were defined as the composite of MACEs - sudden cardiac death, non-fatal myocardial infarction, sustained ventricular arrhythmias, atrial arrhythmias, and hospitalization for heart failure. Results: Patients with severe AS had lower GLS, and increased levels of both biomarkers compared to the control group. Both biomarkers correlated to echocardiographic parameters such as left ventricular (LV) mass index, relative wall thickness, GLS, as well as with the 6-minute walk test distance, and glomerular filtration rate (eGFR). GLS and NT-proBNP predicted MACEs reasonably well, while Galectin-3 did not, after adjustments for confounding factors. Kaplan-Meier analysis showed that the probability of freedom from MACEs was significant in patients who exhibited lower GLS [HR 2.73 (95\% CI 1.01-7.53), $\mathrm{p}<0.05$ ] and higher levels of NT-proBNP [HR 5.22 (95\% CI 1.8514.51), $\mathrm{p}=0.001]$. Conclusions: Among tested parameters, GLS and NT-proBNP were the most reliable predictors of MACEs in patients with severe AS, while Galectin-3 performed more poorly.
\end{abstract}

Keywords: severe aortic stenosis; major cardiovascular adverse events; galectin-3; global longitudinal strain

\section{Introduction}

Aortic stenosis (AS) is nowadays the most frequent valvular heart disease [1]. Although the management of AS has improved over the years with the development of more advanced imaging techniques and novel therapeutic procedures such as transcatheter aortic valve implantation (TAVI), the pathophysiology of AS is not fully understood.

Received 30.01.2018 Accepted 02.03.2018

Med Ultrason

2018, Vol. 20, No 2, 205-212

Corresponding author: Lucia Agoston-Coldea

"Iuliu Hatieganu" University of Medicine and Pharmacy

2-4 Clinicilor street, 400006, Cluj-Napoca,

Romania

Phone: +40264591942 ; Fax: +40264599817

E-mail: luciacoldea@yahoo.com
Inflammation and fibrosis are involved in the progression of AS, occurring in both the leaflets and the myocardium due to prolonged exposure to pressure overload [2]. As myocardial fibrosis develops, the left ventricular (LV) diastolic and systolic function become impaired, leading to poorer clinical outcomes including lower long-term survival after aortic valve replacement $[3,4]$.

Current guidelines recommend aortic valve replacement in severe AS patients who are symptomatic, have impaired left ventricular ejection fraction (LVEF) $<50 \%$ or an abnormal exercise test [5]. However, LVEF is not always reliable, as the reported inter- and intra-observer variability can be as high as $10 \%[6,7]$, and may only become impaired in the late stages of the disease. By contrast, LV global longitudinal strain (GLS) determined by speckle tracking is highly reproducible, and occurs before LVEF impairment in patients with severe AS [8]. 
Considering the contribution of inflammation and fibrosis in the progression of AS, the study of biomarkers might also improve patient management. Among novel biomarkers of myocardial fibrosis and inflammation, Galectin-3 has recently gained interest. Galectin-3 is a $\beta$-galactoside binding lectin which is upregulated in patients with heart failure [9]; it has been identified in valvular tissue explanted from patients with AS who underwent aortic valve replacement surgery [10]. Recent studies have shown that elevated Galectin-3 levels predicted poor outcomes after TAVI [11], suggesting that it may be of use in the management of these patients.

The evolution of severe AS is often complicated by major adverse cardiovascular events (MACEs), including sudden cardiac death, non-fatal myocardial infarction, sustained ventricular arrhythmias, atrial arrhythmias, and hospitalization for heart failure. Several parameters were shown to have predictive values for such events. A reduced GLS predicted cardiovascular mortality and hospitalization for worsening heart failure with higher accuracy than a combined predefined model including the EuroScore, known ischemic heart disease, and LVEF [12]. Among biomarkers of heart failure, N-terminal proB-type natriuretic peptide (NT-proBNP) was proved to predict cardiovascular death in severe AS patients [13], and contribute to risk stratification. However, NT-proBNP levels do not reflect on-going chronic inflammation.

Considering the involvement of Galectin-3 in the pathophysiology of AS and inflammation, in the current study we aimed to test the predictive ability for MACEs of both GLS and Galectin-3, in addition to NT-proBNP, in patients with severe AS.

\section{Material and Methods}

\section{Study patients and design}

We conducted a prospective study on 42 consecutive patients with severe AS who were examined in the $2^{\text {nd }}$ Department of Internal Medicine of the Iuliu Hatieganu University of Medicine and Pharmacy, Cluj-Napoca, Romania between March 2016 and March 2017. Severe AS was defined as 1) peak aortic jet velocity $\geq 4 \mathrm{~m} / \mathrm{s}$, and/or 2) mean transvalvular gradient through the aortic valve $\geq 40$ $\mathrm{mmHg}$, and/or 3) aortic valve area (AVA) $\leq 1.0 \mathrm{~cm}^{2}$ (or AVA indexed to body surface area $\left.\leq 0.6 \mathrm{~cm}^{2} / \mathrm{m}^{2}\right)[5,14]$. Patients with other moderate or severe valvular disease, rheumatic or post-irradiation AS, history of coronary artery disease, prior valve replacement surgery, current inflammatory or infectious diseases, known active neoplasia, end-stage renal disease on dialysis, cirrhosis, pulmonary fibrosis, poor echocardiographic window or those who refused to participate in the research were excluded.
The 42 patients in the test group were compared against 42 controls, matched for age and sex. Patients in the control group had no chronic condition except arterial hypertension, symptoms, normal clinical examination, and normal tests results.

The current research was approved by the local Ethics Committee. All patients and controls signed an informed consent before being enrolled. The study was performed in compliance with the Declaration of Helsinki.

\section{Clinical assessment}

We recorded the medical history and cardiovascular risk factors of all enrolled subjects (history of smoking, hypertension, hypercholesterolemia, diabetes, obesity). Coronary artery disease was defined as history of myocardial infarction, angina pectoris, or previous revascularization (by either angioplasty or coronary artery by-pass). Dyspnea was graded according to the New York Heart Association (NYHA) functional classification. Patients with AS were considered asymptomatic if they did not exhibit dyspnea, angina, and/or syncope. A 12-lead electrocardiogram was recorded at enrollment and 24-hour Holter monitoring was performed. Exercise capacity was tested by trained technicians using the 6MWT according to American Thoracic Society guidelines [15]; and the 6-minute walk test distance (6MWD) was recorded.

The risk of mortality after cardiac surgery was assessed by the EuroScore II [16], using the on-line software available onwww.euroscore.org.

\section{Biochemical analysis}

Two peripheral venous blood samples were collected after at least 12 hours of fasting. One blood sample was used for standard biochemistry tests, performed on a Beckman Coulter AU480 Chemistry Analyzer and for determininghs CRP levels by the Sandwich-ELISA method (KAPDB4360 kit, DIA source ImmunoAssays SA, Belgium). The second blood sample was centrifuged and serum was stored at $-80^{\circ} \mathrm{C}$ until the end of the study and used for measuring Galectin-3 and NT-proBNP levels. We used Human GAL3 (Galectin-3) ELISA Kits, Elabscience Biotechnology Co., Ltd kits for Galectin-3 and Sandwich-ELISA kits, Elabscience Biotechnology Co., Ltd for NT-proBNP. Measurements were performed according to the specifications of the manufacturers by a single investigator who was blinded to all other data.

Glomerular filtration rate (eGFR), was estimated by the Chronic Kidney Disease Epidemiology Collaboration equation [17]. Renal function was considered impaired at $\mathrm{eGFR}<60 \mathrm{~mL} / \mathrm{min} / 1.73 \mathrm{~m}^{2}$.

\section{Echocardiography}

Transthoracic echocardiography (TTE) was performed using a General Electric Vivid E9 (GE Health Medical, Horten, Norway) echocardiograph with aM5S 
$1.5 / 4.6 \mathrm{MHz}$ active matrix phased array transducer. $\mathrm{Pa}$ tients and volunteers in the control group were examined by two investigators working in consensus, with more than 10 years of experience in echocardiography. Investigators were blinded to all clinical and laboratory data.

The severity of AS was graded by peak aortic flow velocity, mean transaortic gradients, and AVA indexed to body surface area (BSA) [18]. LV remodelling was quantified by wall thickness, and LV end-diastolic and endsystolic diameters and volumes, indexed to BSA; left ventricular mass (LVM) calculated by the Devereux formula, and relative wall thickness (RWT) was calculated as $2 \times L V$ posterior wall thickness internal LV diameter in diastole. In patients with increased LV mass, concentric hypertrophy was defined as RWT $>0.42$ and eccentric hypertrophy as RWT $\leq 0.42$ [19].

LVEF was determined by the bi-plane Simpson's modified rule [19]. LV diastolic function and filling pressures were assessed according to the recommendations of the American Society of Echocardiography and the European Association of Cardiovascular Imaging [20].

GLS was determined by 2D speckle tracking from the apical 2-, 3- and 4-chamber views based on the American Society of Echocardiography's 17-segment LV model. In each of the apical views, three sampling points were manually placed on the endocardial border of the septal and lateral mitral annulus, and at the apex. The region of interest was automatically generated and manually edited, aiming at optimum endocardial and epicardial border tracing [21]. Representative cardiac cycles were chosen to determine longitudinal strain, aiming to achieve the best tracking of the myocardium and the most visually satisfying strain curves. Premature beats were excluded. Aortic valve closure was visually identified from the apical long axis view. GLS was calculated as the average of regional strains. The available automated function imaging software package was used for analysis. The software only allowed the calculation of GLS if tracking was adequate in at least five of six segments in each apical view. If more than three segments were not adequately tracked, GLS analysis was not performed.

Right chamber size and function and the probability of pulmonary hypertension were assessed following European Society of Cardiology Guidelines [19,22].

\section{Clinical outcomes}

Patients were followed-up over a mean interval of 347 days (range 60-450 days) by hospital visits, telephone house-calls, or both. We monitored the occurrence of MACEs - sudden cardiac death, myocardial infarction, sustained ventricular arrhythmias, atrial arrhythmias, and hospitalization for heart failure. Hospitalizations due to non-cardiac causes were not considered. The last evalu- ation of patient survival status was performed in May 2017 (the follow-up closing date).

\section{Statistical analysis}

The normal distribution of data was tested using the Kolmogorov-Smirnoff test for continuous variable distribution. Data with parametric distribution were reported as mean and standard deviation (SD), while data with non-parametric distribution were reported as median and interquartile range (IQR). Relative frequencies for categorical variables were reported as percentages. Comparisons between the test and the control groups were made by the Student's $t$ test for data with Gaussian distribution and the Mann-Whitney test for data with non-Gaussian distribution. Univariable and stepwise multivariable logistic regression analysis was used to identify determinants of Galectin-3, NT-proBNP and GLS. Multivariate Cox proportional hazards regression analysis was performed to detect independent predictors of MACEs. Event-free survival (time to first event) was generated by the Kaplan-Meier method and statistical significance was determined by the log-rank test. Receiver operating characteristic (ROC) curve analysis was performed to study the predictive ability of GLS and biomarkers for MACEs $p$ value $<0.05$ was considered statistically significant. The statistical analysis was performed using MedCalc Software, version 16.1.2 (Mariakerke, Belgium).

\section{Results}

Among the 42 AS patients, 36 underwent surgery for aortic valve replacement. Three other patients refused or postponed surgery, while three were minimally symptomatic and were not scheduled for aortic valve replacement until the end of the study.

The demographic data, clinical characteristics, laboratory data, and echocardiographic findings are detailed in Table I. Patients in the AS group had significantly higher body mass index, and were more often dyslipidemic, active smokers or hypertensive, although no statistically significant differences were recorded between the two groups in terms of systolic and diastolic blood pressures. Differences occurred between the two groups in Galectin-3, NT-proBNP and hsCRP levels, which were significantly higher in AS patients compared with the control group.

In AS patients, the etiology of the valvular disease was calcification of a trileaflet valve $(n=34)$, bicuspid aortic valve $(n=4)$, rheumatic disease $(n=2)$, or undetermined $(n=2)$. The AVA index ranged between 0.40 and $0.60 \mathrm{~cm}^{2} / \mathrm{m}^{2}$. The peak and mean aortic pressure gradients were 74.0 (11.3) $\mathrm{mmHg}$ and 45.1 (13.0) $\mathrm{mmHg}$, respectively. 
Table I. Baseline demographic characteristics and echocardiography parameters in patients with severe aortic stenosis vs. the control group

\begin{tabular}{|c|c|c|c|}
\hline Clinical characteristics & Severe AS group $(n=42)$ & Control group $(n=42)$ & p-Value \\
\hline Age, years & $73(6)$ & $73(8)$ & NS \\
\hline Male sex, n (\%) & $23(54.8)$ & $23(54.8)$ & NS \\
\hline Body surface area, $\mathrm{m}^{2}$ & $1.88(0.21)$ & $1.86(0.18)$ & NS \\
\hline Body-mass index, $\mathrm{kg} / \mathrm{m}^{2}$ & $27.9(5.5)$ & $25.1(4.1)$ & $<0.001$ \\
\hline Heart rate, bpm & $67.3(8.6)$ & $67.9(11.6)$ & NS \\
\hline Systolic blood pressure, $\mathrm{mmHg}$ & $138.6(19.4)$ & $133.5(19.1)$ & NS \\
\hline Diastolic blood pressure, $\mathrm{mmHg}$ & $79.7(13.7)$ & $83.5(9.4)$ & NS \\
\hline Hypertension, n (\%) & $29(69)$ & $12(28.5 \%)$ & $<0.001$ \\
\hline Diabetes mellitus, n (\%) & $13(30.9)$ & $10(23.8 \%)$ & NS \\
\hline Dyslipidemia, n (\%) & $28(66.7)$ & $15(35.7 \%)$ & $<0.001$ \\
\hline Smoking, n (\%) & $23(54.3)$ & $16(38.1 \%)$ & $<0.001$ \\
\hline 6MWD, $\mathrm{m}$ & $410(124)$ & $605(93)$ & $<0.001$ \\
\hline Coronary artery disease, $\mathrm{n}(\%)$ & $7(16.7)$ & & \\
\hline Atrial arrhythmia, n (\%) & $11(26.2)$ & & \\
\hline Chronic obstructive lung disease, $\mathrm{n}(\%)$ & $3(7.1)$ & & \\
\hline Peripheral vascular disease, n (\%) & $21(50)$ & & \\
\hline Syncope, n (\%) & $8(19)$ & & \\
\hline Angina, $n(\%)$ & $17(40.5)$ & & \\
\hline NYHA functional class $\geq$ III, n (\%) & $11(26.2)$ & & \\
\hline Logistic EuroScore, \% & $3.4(1.2-5.6)$ & & \\
\hline \multicolumn{4}{|l|}{ Biomarker levels } \\
\hline Galectin-3, ng/mL, IQR & $15.4(2.2-24.4)$ & $4.9(4.1-12.6)$ & $<0.001$ \\
\hline NT-proBNP, pg/mL, IQR & $1797(390-10267)$ & $432(380-620)$ & $<0.001$ \\
\hline hsCRP, pg/mL, IQR & $1.10(0.49-2.55)$ & $0.72(0.51-1.15)$ & $<0.001$ \\
\hline $\mathrm{eGFR}, \mathrm{ml} / \mathrm{min} / 1.73 \mathrm{~m}^{2}$ & $66.6(12.3)$ & $81.3(14.1)$ & $<0.001$ \\
\hline \multicolumn{4}{|l|}{ Medications } \\
\hline$\beta$-blockers, n (\%) & $34(81)$ & $6(14.2)$ & \\
\hline ACEI or ARB, n (\%) & $38(90.5)$ & $8(19)$ & \\
\hline Calcium channel blocker, n (\%) & $5(11.9)$ & $2(4.7)$ & \\
\hline Statins, n (\%) & $35(83.3)$ & $6(14.2)$ & \\
\hline ASA or antiplatelet, $\mathrm{n}(\%)$ & $27(64.3)$ & $7(16.6)$ & \\
\hline Diuretics, n (\%) & $35(83.3)$ & $3(7.1)$ & \\
\hline \multicolumn{4}{|l|}{ Echocardiography } \\
\hline IVSd, mm & $13.4(1.7)$ & $9.2(1.2)$ & $<0.001$ \\
\hline LVPWd, mm & $12.9(3.6)$ & $9.0(0.91)$ & $<0.001$ \\
\hline LVEDD, $\mathrm{mm}$ & $51.5(5.1)$ & $47.4(2.8)$ & NS \\
\hline LVESD, mm & $34.5(6.2)$ & $29.7(2.3)$ & $<0.001$ \\
\hline LVEDV index, $\mathrm{mL} / \mathrm{m}^{2}$ & $73.6(14.2)$ & $68.7(14.3)$ & NS \\
\hline LVESV index, $\mathrm{mL} / \mathrm{m}^{2}$ & $30.7(11.8)$ & $24.7(7.0)$ & $<0.01$ \\
\hline $\mathrm{LVM}$ index, $\mathrm{g} / \mathrm{m}^{2}$ & $95.3(26.9)$ & $66.4(13.9)$ & $<0.001$ \\
\hline LVEF, \% & $57.0(9.6)$ & $64.2(6.7)$ & $<0.01$ \\
\hline RWT & $0.52(0.10)$ & $0.38(0.03)$ & $<0.001$ \\
\hline $\mathrm{LAV}$ index, $\mathrm{mL} / \mathrm{m}^{2}$ & $49.7(11.6)$ & $27.3(6.4)$ & $<0.001$ \\
\hline $\mathrm{E} / \mathrm{E}$ ' ratio & $10.2(3.2)$ & $6.5(0.86)$ & $<0.001$ \\
\hline $\mathrm{DT}, \mathrm{ms}$ & $221(54.5)$ & $185(8.9)$ & $<0.001$ \\
\hline GLS, \% & $-15.6(3.8)$ & $-20.5(0.62)$ & $<0.001$ \\
\hline $\mathrm{sPAP}, \mathrm{mmHg}$ & $42.5(11.7)$ & $21.5(4.7)$ & $<0.001$ \\
\hline TAPSE, $\mathrm{mm}$ & $19.0(3.7)$ & $22.9(2.8)$ & $<0.001$ \\
\hline Peak aortic velocity, $\mathrm{m} / \mathrm{s}$ & $4.4(0.34)$ & $1.3(0.21)$ & $<0.001$ \\
\hline Peak aortic gradient, $\mathrm{mmHg}$ & $74.0(11.3)$ & $6.9(2.1)$ & $<0.001$ \\
\hline Mean aortic gradient, $\mathrm{mmHg}$ & $45.1(13.0)$ & $3.6(0.62)$ & $<0.001$ \\
\hline AVA index, $\mathrm{cm}^{2} / \mathrm{m}^{2}$ & $0.50(0.08)$ & $1.9(0.07)$ & $<0.001$ \\
\hline
\end{tabular}

Data are presented as mean (standard deviation) or median (IQR) or $\mathrm{n}(\%) . \mathrm{n}$, number patients; IQR, interquartile range; NYHA, New York Heart Association; NT-proBNP, N-terminal pro-Brain Natriuretic Peptide; hsCRP, high sensitive C reactive protein; eGFR, estimated glomerular filtration rate; ACEI, angiotensin converting enzyme inhibitor; ARB, angiotensin receptor blocker; ASA, acetylsalicylic acid; IVSd, diastolic interventricular septum thickness; LVPWd, diastolic left ventricular posterior wall thickness; LVEDD, left ventricular end-diastolic diameter; LVESD, left ventricular end-systolic diameter; LVEDV, left ventricular end-diastolic volume; LVESV, left ventricular end-systolic volume; LVM, left ventricular mass; LVEF, left ventricular ejection fraction; RWT, relative wall thickness; LAV, left atrial volume; E, peak mitral flow velocity; E', peak myocardial velocity at the mitral valve annulus; DT, early diastolic filling deceleration time; GLS, global longitudinal strain; sPAP, systolic pulmonary artery pressure; 6MWD, six minute walk distance; TAPSE, tricuspid annular plane systolic excursion; AVA, aortic valve area. 
In the AS group, LV wall thickness was significantly increased compared to the control group, as were the RWT and LV mass, while LVEF was lower. The E/E' ratio was higher in test group patients, also exhibiting a larger LAV index, increased sPAP and lower TAPSE.
Importantly, GLS was significantly lower in AS patients compared with the control group.

We further tested correlations between Galectin-3 and NT-proBNP levels, and significant parameters of AS severity (mean gradients and AVA), as well as indices of

Table II. Correlations between AS patients' characteristics, echocardiography parameters, plasma levels of Galectin-3, and N-terminal pro-B-type natriuretic peptide

\begin{tabular}{lllllllllll}
\hline Characteristic & Age & eGRF & 6MWD & AVA & MPG & LVEF & LVM index & RWT & GLS \\
\hline Galectin-3 & $\mathrm{r}$ & -0.087 & -0.356 & -0.528 & 0.193 & -0.195 & -0.182 & 0.438 & 0.476 & -0.608 \\
& $\mathrm{p}$ & $\mathrm{NS}$ & 0.0009 & $<0.0001$ & NS & NS & NS & $<0.0001$ & $<0.0001$ & $<0.0001$ \\
NT-proBNP & $\mathrm{r}$ & -0.120 & -0.393 & -0.570 & 0.184 & -0.162 & -0.352 & 0.582 & 0.556 & -0.729 \\
& $\mathrm{p}$ & NS & 0.0002 & $<0.0001$ & NS & NS & 0.001 & $<0.0001$ & $<0.0001$ & $<0.0001$ \\
\hline
\end{tabular}

AVA, aortic valve area; eGFR, estimated glomerular filtration rate; GLS, global longitudinal strain; LVEF, left ventricular ejection fraction; LVM, left ventricular mass; MPG, mean transvalvular aortic pressure gradient; 6MWD, six minute walk distance; RWT, relative wall thickness; NT-proBNP, N-terminal pro-B-type natriuretic peptide. Data are shown as Pearson's or Spearman's correlation coefficients (r) for parametric and non-parametric characteristics, respectively, and associated p values. NS, not significant.

Table III. All major adverse cardiovascular events in univariable and multivariable analyses by logistic regression

\begin{tabular}{|c|c|c|c|c|c|c|}
\hline & \multirow{2}{*}{$\begin{array}{l}\text { No events } \\
\mathbf{n}=\mathbf{2 7}\end{array}$} & \multirow{2}{*}{$\begin{array}{l}\text { Events } \\
n=15\end{array}$} & \multicolumn{2}{|c|}{ Univariable analysis } & \multicolumn{2}{|c|}{ Multivariable analysis } \\
\hline & & & $\begin{array}{l}\text { Unadjusted OR } \\
(95 \% \text { CI })\end{array}$ & $\begin{array}{l}\mathbf{p} \\
\text { Value }\end{array}$ & $\begin{array}{l}\text { Adjusted OR } \\
(95 \% \text { CI) }\end{array}$ & $\begin{array}{l}\text { p } \\
\text { Value }\end{array}$ \\
\hline Age, years & $74(6)$ & $72(7)$ & $1.01(0.95-1.07)$ & NS & & \\
\hline Male sex, $n, \%$ & $15(55.5)$ & $8(53.3)$ & $1.64(0.61-4.40)$ & NS & & \\
\hline Body surface area, $\mathrm{m}^{2}$ & $1.89(0.22)$ & $1.88(0.21)$ & $1.28(0.11-14.2)$ & NS & & \\
\hline Systolic blood pressure & $134(20.8)$ & $126(15.9)$ & $1.00(0.98-1.03)$ & NS & & \\
\hline Diastolic blood pressure & $81(14.0)$ & $76(12.8)$ & $1.01(0.97-1.06)$ & NS & & \\
\hline Galectin-3, ng/mL & $15.2(5.1-23.6)$ & $17.7(2.2-24.4)$ & $1.08(0.95-1.82)$ & $<0.01$ & $0.97(0.86-1.13)$ & NS \\
\hline NT-proBNP, pg/mL & $1350(390-8978)$ & $3900(690-10267)$ & $1.02(1.01-1.06)$ & 0.004 & $1.18(1.07-1.42)$ & $<0.01$ \\
\hline hs-CRP, pg/mL & $0.92(0.51-1.87)$ & $1.27(0.49-2.55)$ & $1.20(1.17-1.83)$ & 0.02 & $1.06(0.94-1.14)$ & NS \\
\hline $\mathrm{eGFR}, \mathrm{ml} / \mathrm{min} / 1.73 \mathrm{~m}^{2}$ & $69(11.6)$ & $62(13.4)$ & $0.95(0.89-1.01)$ & NS & & \\
\hline $6 \mathrm{MWD}, \mathrm{m}$ & $445(120)$ & $345(108)$ & $0.99(0.98-1.00)$ & 0.007 & $1.00(0.99-1.01)$ & NS \\
\hline IVSd, mm & $13.3(1.5)$ & $13.7(1.1)$ & $1.13(0.79-1.63)$ & NS & & \\
\hline LVPWd, mm & $12.7(1.7)$ & $13.2(1.2)$ & $1.12(0.81-1.68)$ & NS & & \\
\hline LVEDD, mm & $51.1(4.3)$ & $52.3(6.2)$ & $1.07(0.96-1.20)$ & NS & & \\
\hline LVESD, mm & $34.1(5.1)$ & $35.4(7.8)$ & $1.04(0.95-1.14)$ & NS & & \\
\hline LVEDV index, $\mathrm{mL} / \mathrm{m}^{2}$ & $71.9(11.2)$ & $76.6(18.5)$ & $1.00(0.96-1.03)$ & NS & & \\
\hline LVESV index, $\mathrm{mL} / \mathrm{m}^{2}$ & $27.9(7.1)$ & $35.6(16.4)$ & $0.97(0.91-1.02)$ & $<0.01$ & $0.98(0.95-1.03)$ & NS \\
\hline LVM index, $\mathrm{g} / \mathrm{m}^{2}$ & $90.1(19.4)$ & $104.8(20.7)$ & $1.01(0.99-1.03)$ & NS & & \\
\hline LVEF, \% & $56.5(6.4)$ & $52.4(5.1)$ & $1.04(0.98-1.11)$ & NS & & \\
\hline RWT & $0.54(0.09)$ & $0.43(0.11)$ & $2.12(1.10-2.96)$ & $<0.01$ & $1.06(1.02-1.11)$ & NS \\
\hline LAV index, $\mathrm{mL} / \mathrm{m}^{2}$ & $47.2(11.2)$ & $51.3(6.7)$ & $0.96(0.84-1.10)$ & NS & & \\
\hline GLS, \% & $-16.4(1.7)$ & $-14.9(3.7)$ & $0.69(0.49-0.97)$ & 0.009 & $1.04(1.01-1.13)$ & $<0.01$ \\
\hline $\mathrm{E} / \mathrm{E}$ ' ratio & $9.3(2.6)$ & $11.8(3.6)$ & $1.28(1.03-1.61)$ & $<0.01$ & $0.98(0.87-0.99)$ & NS \\
\hline Peak aortic velocity, m/s & $4.3(0.15)$ & $4.5(0.51)$ & $2.48(0.57-10.7)$ & NS & & \\
\hline Peak aortic gradient, $\mathrm{mmHg}$ & $72.2(15.1)$ & $77.1(13.4)$ & $1.02(0.98-1.08)$ & NS & & \\
\hline Mean aortic gradient, $\mathrm{mmHg}$ & $43.1(12.8)$ & $49.4(14.5)$ & $1.02(0.97-1.06)$ & NS & & \\
\hline AVA index, $\mathrm{cm}^{2} / \mathrm{m}^{2}$ & $0.51(0.09)$ & $0.49(0.08)$ & $1.38(0.67-3.93)$ & NS & & \\
\hline
\end{tabular}

n, number patients; NT-proBNP, N-terminal pro-Brain Natriuretic Peptide; hsCRP, high sensitive C reactive protein; eGFR, estimated glomerular filtration rate; IVSd, diastolic interventricular septum thickness; LVPWd, diastolic left ventricular posterior wall thickness; LVEDD, left ventricular end-diastolic diameter; LVESD, left ventricular end-systolic diameter; LVEDV, left ventricular end-diastolic volume; LVESV, left ventricular end-systolic volume; LVM, left ventricular mass; LVEF, left ventricular ejection fraction; RWT, relative wall thickness; LAV, left atrial volume; E, peak mitral flow velocity; E', peak myocardial velocity at the mitral valve annulus; DT, early diastolic filling deceleration time; GLS, global longitudinal strain; sPAP, systolic pulmonary artery pressure; TAPSE, tricuspid annular plane systolic excursion; AVA, aortic valve area; 6MWD, six minute walk distance; NS, not significant. 
LV remodelling and dysfunction - RWT, LVM index, LVEF and GLS. There were no correlations between the echocardiographic parameters of AS severity and any of the tested biomarkers. Both Galectin-3 and NT-proBNP levels were, however, positively correlated to the LVM index, RWT and negatively to GLS. NT-proBNP correlated significantly and inversely to LVEF, while Galectin-3 levels did not. Correlations between NT-proBNP levels and LVEF were only weak, despite reaching statistical significance. Both biomarkers correlated negatively to 6MWDs and to eGFR (Table II).

Among the 42 patients with AS, 15 had MACEs: death $(\mathrm{n}=1)$ non-fatal myocardial infarction $(\mathrm{n}=1)$, sustained ventricular arrhythmias $(n=1)$, atrial arrhythmias $(n=5)$, and hospitalization for heart failure $(\mathrm{n}=10)$. Univariable analysis by logistic regression yielded statistically significant direct correlations between Galectin-3, NT-proBNP and CRP levels and the occurrence of MACEs. Increased LV end-systolic volume, RWT, E/E' ratio and impaired GLS were also associated with MACEs. In multivariable analysis, only the correlations to NT-proBNP levels and GLS remained statistically significant (Table III).

Kaplan-Meier analysis assessing correlations between GLS $<-14.9 \%$ and NT-proBNP $>3900 \mathrm{pg} / \mathrm{mL}$ and overall MACEs occurrence, showed that the probability of freedom from MACEs was significant in patients who exhibited lower GLS [HR $2.73(95 \%$ CI 1.01-7.53), $\mathrm{p}<0.05]$ and
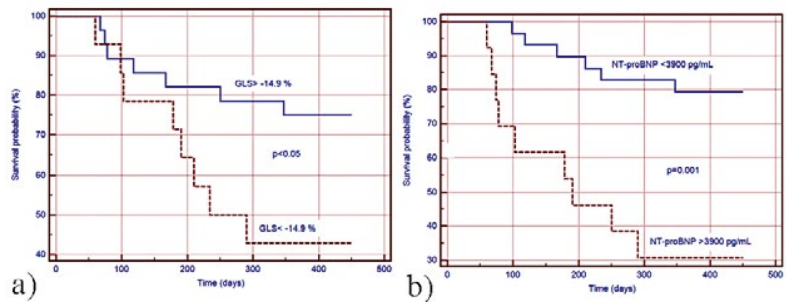

Fig 1. The Kaplan-Meier event-free survival curves according to the following categorical variables which were selected in the multivariate model to predict outcomes in patients with AS: a) left ventricular global longitudinal strain (GLS); b) N-terminal pro-B-type natriuretic peptide (NT-proBNP). high levels NT-proBNP [HR 5.22 (95\% CI 1.85-14.51), $\mathrm{p}=0.001$ ] (fig 1). Galectin-3 levels were not predictive of MACEs [HR 2.69 (95\% CI 0.89-8.13), $\mathrm{p}<0.05$ ].

The predictive power of GLS for identifying patients with MACEs was slightly lower than that of NT-proBNP, but higher than that of Galectin-3 or other echocardiographic parameters such as LVEF, LVM index, or RWT (Table IV).

\section{Discussions}

In the current study, we aimed to assess the diagnostic and prognostic ability of GLS and Galectin-3 in patients with severe AS. Among echocardiography parameters, GLS performed best, having a higher sensitivity than the LVEF, LVM index, and RWT for identifying patients with cardiovascular events: the specificity was however, lower than that of LVEF.

The high sensitivity of GLS was confirmed in previous studies; GLS was shown to detect early systolic dysfunction [8,23], to associate with MACEs [24], and provide incremental value to clinical parameters, including the EuroScore, for predicting mortality in AS patients with preserved [25] or mildly impaired LVEF [12]. Moreover, Gelsomino et al showed that, in patients with AS and preserved LVEF, GLS could accurately predict LVM regression after aortic valve replacement [26].

Although Galectin-3 levels were significantly higher in patients with AS than in controls, and correlated moderately to echocardiographic parameters of LV remodelling such as LVM index and RWT, and strongly to GLS, it failed to accurately predict MACEs. After adjustment for confounding factors in multivariate analysis, it no longer correlated with MACEs, while GLS and NT-proBNP did.

Our results are consistent with previously published data. Although Galectin-3 is known to be involved in inflammation and the development of fibrosis [4], its clinical use is hindered by the fact that it is not specific for identifying myocardial fibrosis. In our study, as well as in previously published research, confounding factors

Table IV. Ability of the biochemistry and echocardiography parameters for predicting outcome

\begin{tabular}{lllllll}
\hline Variables & Sensibility & Specificity & PPV & PNV & ROC threshold & AUC \\
\hline LVEF & 0.66 & 0.92 & 0.88 & 0.75 & $<52$ & 0.662 \\
LVM index & 0.69 & 0.70 & 0.67 & 0.76 & $>97$ & 0.616 \\
RWT & 0.80 & 0.73 & 0.74 & 0.85 & $<0.41$ & 0.754 \\
GLS & 0.82 & 0.76 & 0.72 & 0.86 & $<-14.9$ & 0.803 \\
Galectin-3 & 0.56 & 0.82 & 0.58 & 0.73 & $>17.7$ & 0.641 \\
NT-proBNP & 0.87 & 0.80 & 0.72 & 0.90 & $>3900$ & 0.894 \\
\hline
\end{tabular}

LVEF, left ventricular ejection fraction; LVM, left ventricular mass; RWT, relative wall thickness; GLS, global longitudinal strain; NTproBNP, N-terminal pro-Brain Natriuretic Peptide; PPV, predictive positive value; PNV, predictive negative value; ROC receiver operating characteristic curve analysis; AUC, area under curve. 
have emerged, mostly linked to the presence of inflammation and fibrosis in other organs. Among confounders, impaired renal function is particularly prominent [27,28], which is an important issue considering the fact that many patients with cardiovascular disease, particularly heart failure, evidence associate kidney dysfunction [29]. In the research by Bobrowska et al, Galectin-3 levels were weakly associated with medium-term mortality in patients undergoing surgical or interventional treatment of severe AS, but the correlation was lost after adjustment for eGFR. Galectin-3 levels $>17.8 \mathrm{ng} / \mathrm{mL}$ only predicted mortality in the subgroup treated by balloon aortic valvuloplasty, even after adjustment for eGFR and valvulo-arterial impedance [28]. Interestingly, Baldenhofer et al [11] reported that Galectin-3 predicted outcomes after TAVI independently of age, sex, glomerular filtration rate, and NT-proBNP [11]. In a recent study Galectin-3 was not associated with either AS severity, or functional status, and did not provide prognostic information on the occurrence of AS-related events [27].

Unlike Galectin-3, NT-proBNP performed well, yielding the most reasonable sensitivity and specificity for the occurrence of MACEs. This is consistent with data from previous studies. In a series of 1953 consecutive patients with at least moderate AS, BNP clinical activation, defined as measured $\mathrm{BNP} / \mathrm{age}$ was associated with higher longterm mortality [30]. Moreover, BNP levels, particularly in combination with echocardiographic markers of LV dysfunction, were shown to anticipate the development of symptoms in asymptomatic patients with severe AS [31] and to predict clinical outcomes [32] in such patients.

It is possible that, in the future, BNP or NT-proBNP, as well as echocardiographic markers of LV function impairment such as GLS, might contribute to the selection of asymptomatic AS patients for early surgery. The role of Galectin-3 in the management and prognostic assessment of AS patients remains, however, uncertain.

The current study has some limitations generated by the rather low number of patients and the fact that patient follow-up was limited to as little as 60 days for the last enrolled subjects. Further, investigations by echocardiography and Galectin-3 levels were only performed at enrollment and were not repeated afterwards by the investigators involved in the current study. Further research on a larger number of patients and with an extended period of follow-up is needed to endorse the results of the current study.

\section{Conclusions}

In our study, the best predictors of MACEs in patients with severe AS undergoing surgery were NT-proBNP levels and reduced GLS. Although Galectin-3 levels correlated well with echocardiographic parameters of LV remodelling and dysfunction, it did not predict MACEs after adjustment for confounding factors.

Funding: This work was supported by grant 4994/1/2016, "Iuliu Hatieganu" University of Medicine and Pharmacy of Cluj-Napoca, Romania.

\section{Conflict of interest: none}

\section{References}

1. Iung B, Baron G, Butchart EG, et al. A prospective survey of patients with valvular heart disease in Europe: The Euro Heart Survey on Valvular Heart Disease. Eur Heart J 2003;24:1231-1243.

2. Chambers J. The left ventricle in aortic stenosis: evidence for the use of ACE inhibitors. Heart 2006;92:420-423.

3. Milano AD, Faggian G, Dodonov M, et al. Prognostic value of myocardial fibrosis in patients with severe aortic valve stenosis. J Thorac Cardiovasc Surg 2012;144:830-837.

4. Azevedo CF, Nigri M, Higuchi ML, et al. Prognostic significance of myocardial fibrosis quantification by histopathology and magnetic resonance imaging in patients with severe aortic valve disease. J Am Coll Cardiol 2010;56:278-287.

5. Vahanian A, Alfieri O, Andreotti F, et al ; Joint Task Force on the Management of Valvular Heart Disease of the European Society of Cardiology (ESC); European Association for Cardio-Thoracic Surgery (EACTS). Guidelines on the management of valvular heart disease (version 2012). Eur Heart J 2012;33:2451-2496.

6. Thavendiranathan P, Grant AD, Negishi T, Plana JC, Popović ZB, Marwick TH. Reproducibility of echocardiographic techniques for sequential assessment of left ventricular ejection fraction and volumes: application to patients undergoing cancer chemotherapy. J Am Coll Cardiol 2013;61:77-84.

7. Galema TW, Geleijnse ML, Yap SC, et al. Assessment of left ventricular ejection fraction after myocardial infarction using contrast echocardiography. Eur J Echocardiogr 2008;9:250-254.

8. Delgado V, Tops LF, van Bommel RJ, et al. Strain analysis in patients with severe aortic stenosis and preserved left ventricular ejection fraction undergoing surgical valve replacement. Eur Heart J 2009;30:3037-3047.

9. Lopez-Andres N, Rossignol P, Iraqi W, et al. Association of galectin-3 and fibrosis markers with long-term cardiovascular outcomes in patients with heart failure, left ventricular dysfunction, and dyssynchrony: insights from the CAREHF (Cardiac Resynchronization in Heart Failure) trial. Eur J Heart Fail 2012;14:74-81.

10. Sádaba JR, Martínez-Martínez E, Arrieta V, et al. Role for Galectin-3 in Calcific Aortic Valve Stenosis. J Am Heart Assoc 2016;5:e004360. 
11. Baldenhofer G, Zhang K, Spethmann S, et al. Galectin-3 predicts short- and long-term outcome in patients undergoing transcatheter aortic valve implantation (TAVI). Int $\mathrm{J}$ Cardiol 2014;177:912-917.

12. Dahl JS, Videbæk L, Poulsen MK, Rudbæk TR, Pellikka PA, Møller JE. Global strain in severe aortic valve stenosis: relation to clinical outcome after aortic valve replacement. Circ Cardiovasc Imaging 2012;5:613-620.

13. Lim P, Monin JL, Monchi M, et al. Predictors of outcome in patients with severe aortic stenosis and normal left ventricular function: role of B-type natriuretic peptide. Eur Heart J 2004;25:2048-2053.

14. Nishimura RA, Otto CM, Bonow RO, et al. 2014 AHA/ ACC Guideline for the Management of Patients With Valvular Heart Disease: executive summary: a report of the American College of Cardiology/American Heart Association Task Force on Practice Guidelines. Circulation 2014;129:2440-2492.

15. ATS Committee on Proficiency Standards for Clinical Pulmonary Function Laboratories. ATS statement: guidelines for the six-minute walk test. Am J Respir Crit Care Med 2002;166:111-117.

16. Paparella D, Guida P, Di Eusanio G, et al. Risk stratification for in-hospital mortality after cardiac surgery: external validation of EuroSCORE II in a prospective regional registry. Eur J Cardiothorac Surg 2014;46:840-848.

17. Levey AS, Stevens LA, Schmid CH, et al. A new equation to estimate glomerular filtration rate. Ann Intern Med 2009; 150:604-612.

18. Baumgartner H, Hung J, Bermejo J, et al; American Society of Echocardiography; European Association of Echocardiography. Echocardiographic assessment of valve stenosis: EAE/ASE recommendations for clinical practice. J Am Soc Echocardiogr 2009;22:1-23.

19. Lang RM, Badano LP, Mor-Avi V, et al. Recommendations for cardiac chamber quantification by echocardiography in adults: an update from the American Society of Echocardiography and the European Association of Cardiovascular Imaging. J Am Soc Echocardiogr 2015;28:1-39.

20. Nagueh SF, Smiseth OA, Appleton CP, et al. Recommendations for the Evaluation of Left Ventricular Diastolic Function by Echocardiography: An Update from the American Society of Echocardiography and the European Association of Cardiovascular Imaging. J Am Soc Echocardiogr 2016;29:277-314.

21. Voigt JU, Pedrizzetti G, Lysyansky P, et al. Definitions for a common standard for $2 \mathrm{D}$ speckle tracking echocardiography: consensus document of the EACVI/ASE/Industry Task Force to standardize deformation imaging. Eur Heart J Cardiovasc Imaging 2015;16:1-11.

22. Galiè N, Humbert M, Vachiery JL, et al. 2015 ESC/ERS Guidelines for the diagnosis and treatment of pulmonary hypertension: The Joint Task Force for the Diagnosis and Treatment of Pulmonary Hypertension of the European Society of Cardiology (ESC) and the European Respiratory Society (ERS): Endorsed by: Association for European Paediatric and Congenital Cardiology (AEPC), International Society for Heart and Lung Transplantation (ISHLT). Eur Heart J 2016;37:67-119.

23. Cho EJ, Park SJ, Kim EK, et al. Effects of increased left ventricular wall thickness on the myocardium in severe aortic stenosis with normal left ventricular ejection fraction: Two- and three-dimensional multilayer speckle tracking echocardiography. Echocardiography 2017;34:511-522.

24. Nagata Y, Takeuchi M, Wu VC, et al. Prognostic value of LV deformation parameters using 2D and 3D speckle-tracking echocardiography in asymptomatic patients with severe aortic stenosis and preserved LV ejection fraction. JACC Cardiovasc Imaging 2015;8:235-245.

25. Kusunose K, Goodman A, Parikh R, et al. Incremental prognostic value of left ventricular global longitudinal strain in patients with aortic stenosis and preserved ejection fraction. Circ Cardiovasc Imaging 2014;7:938-945.

26. Gelsomino $\mathrm{S}$, Lucà $\mathrm{F}$, Parise $\mathrm{O}$, et al. Longitudinal strain predicts left ventricular mass regression after aortic valve replacement for severe aortic stenosis and preserved left ventricular function. Heart Vessels 2013;28:775-784.

27. Arangalage D, Nguyen V, Robert T, et al. Determinants and prognostic value of Galectin-3 in patients with aortic valve stenosis. Heart 2016;102:862-868.

28. Bobrowska B, Wieczorek-Surdacka E, Kruszelnicka O, Chyrchel B, Surdacki A, Dudek D. Clinical Correlates and Prognostic Value of Plasma Galectin-3 Levels in Degenerative Aortic Stenosis: A Single-Center Prospective Study of Patients Referred for Invasive Treatment. Int J Mol Sci 2017;18:947.

29. Heywood JT, Fonarow GC, Costanzo MR, et al; ADHERE Scientific Advisory Committee and Investigators. High prevalence of renal dysfunction and its impact on outcome in 118,465 patients hospitalized with acute decompensated heart failure: a report from the ADHERE database. J Card Fail 2007;13:422-430.

30. Clavel MA, Malouf J, Michelena HI, et al. B-type natriuretic peptide clinical activation in aortic stenosis: impact on long-term survival. J Am Coll Cardiol 2014;63:2016-2025.

31. Henri C, Dulgheru R, Magne J, et al. Impact of Serial BType Natriuretic Peptide Changes for Predicting Outcome in Asymptomatic Patients with Aortic Stenosis. Can J Cardiol 2016;32:183-189.

32. Lancellotti P, Moonen M, Magne J, et al. Prognostic effect of long-axis left ventricular dysfunction and B-type natriuretic peptide levels in asymptomatic aortic stenosis. Am J Cardiol 2010;105:383-388. 